June - 2008

\title{
Editorial: Ideas whose time has come back
}

\author{
Jon Baggaley \\ Professor of Educational Technology \\ Athabasca University - Canada’s Open University
}

This issue of IRRODL contains papers from Brazil, Greece, Sri Lanka, Canada and the US, and reviews of distance education developments in Africa, Asia, and the Caribbean. A new world of distance education (DE) is coming together in the developing world, as old media such as radio and the telephone merge with each other and the Internet to form wholly original interactive partnerships. So I now offer you a new piece of jargon, coined to pay respect to the creative return to older DE media: 'paradigm rollback' - you heard it here first! This odious but quite typical piece of verbiage signifies "a shift back to an idea whose time has come," as in the case of DE technologies supplanted for a while by the promise that the Internet would do the job better. I just looked the term up on Google, and find only one reference to it so far - in the conference presentation where I coined the term a week ago! Let's see how many references there are to 'paradigm rollback' a few months from now among those for whom jargon is all . . .

The first paper by Stella Porto and Zane Berge discusses how DE is shaped by the socio-political context. Brazil is a developing country undergoing rapid globalization, yet much of its legislation remains rooted in past practices. Porto and Berge argue that legislation in Brazil is currently inimical to the deployment of public DE throughout the country. Private sector companies have stepped in to fill the void created by this problem, in the effort to serve employees' training needs. The downside of this trend, Porto and Berge argue, is that private sector efforts will widen the digital divide between Brazil's urban/ rural and rich/ poor.

The socio-political theme is also pursued by Gerasimos Koustourakis, Chris Panagiotakopoulos, and Dimitris Vergidis in a study employing a Bersteinian theoretical framework at The Hellenic Open University (HOU). The work is grounded within the larger Greek geopolitical landscape, by showing that HOU was born with one foot in the DE world and the other in the traditional educational environment, and that this bifurcated approach is being used to inform other universities' DE practices throughout Greece. The authors argue that it may not be wise to use the HOU as an exemplar at this juncture, owing to the scant research so far conducted into the efficacy of its DE practices.

The remaining main section papers are from North America. Sherry Piezon and William Ferree discuss Perceptions of Social Loafing in Online Learning Groups. The witty and thoughtprovoking concept of social loafing points to an entirely different form of online behaviour compared with that commonly described as 'lurking.' The paper discusses how students' 'loafing' can affect the process and outcomes of online learning, especially in group contexts. It is followed by a study of Online Self-Regulatory Learning Behaviors by Lucy Barnard, Valerie Paton and William Lan. These authors discuss how positive perceptions of online course communication and collaboration have been associated in the literature with better learning 
outcomes, while self-regulated learning behaviors have been linked to academic achievement. Their results, however, indicate that the actual picture is not that simple.

In a related paper, Arbaugh examines and validates the popular Community of Inquiry (CoI) framework for online education, and presents empirical evidence of CoI's ability to predict both perceived learning and satisfaction with the online delivery medium.

We then have a report by Terry Müller who examines the persistence of women as online learners in degree-completion programmes at a college in the Northeastern United States. Müller's study indicates the many barriers to online learning encountered by women, and the gap between this reality and the promise that online university-level courses would provide them with increased educational access, flexibility, and convenience. The paper's constructive finding is that women's persistence in online learning can be increased by efforts to boost their motivation levels in relation to particular personal attributes.

One such attribute, 'emotional intelligence' (EI), is then discussed by Robin Berenson, Gary Boyles, and Anne Weaver, in a study of the relationship between students' EI and final grade point averages. The study indicates that EI profiles can help to predict the students who will become successful online learners, and that 'soft EI skills' can enhance academic success.

The final paper by Gabriella Brandes and Natasha Boskic indicates that the online 'e-portfolio' activity has particular potential for increasing student motivation, satisfaction, and achievement. This Canadian study demonstrates that e-portfolios can be valuable not only for compiling students' learning activities, but also in a central repository that students can use to support one another's learning and to develop collective expertise.

The final sections of this issue contain a Research Notes report from the Open University of Sri Lanka about Instructional Strategies in Teaching Engineering at a Distance.

We then have two book reviews on New Directions in University Education: Perspectives from the Caribbean, and Collaborative Learning: Two perspectives on theory and practice.

The latest installments in the popular Technical Reports concern The Return of Educational Radio, and Mobile Learning in Developing Nations. As at the beginning of this editorial, we see the timeliness and validity of the new 'paradigm rollback' concept!

This typically large edition of IRRODL ends with re-broadcasts of recent sessions organised by the Canadian Institute of Distance Education Research. Over to you!

Jon Baggaley, Guest Editor.
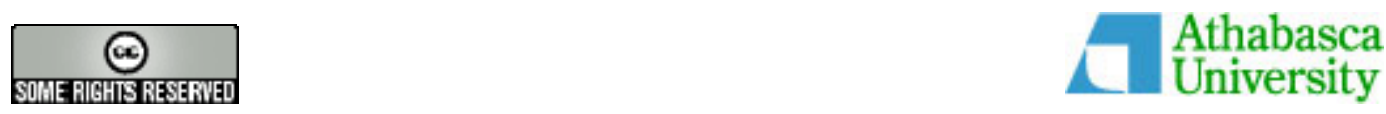